\title{
Steering and focusing effects in TESLA cavity due to high order mode and input couplers
}

\author{
P. Piot, Fermi National Accelerator Laboratory, Batavia, IL 60510, USA, \\ M. Dohlus, K. Flöttmann, M. Marx, S.G. Wipf, \\ Deutsches Elektronen Synchrotron DESY, Hamburg, D-22603 Germany.
}

\begin{abstract}
Many state-of-art electron accelerator proposals incorporate TESLA-type superconducting radio-frequency (rf) cavities [1]. These standing wave rf cavities include rf input couplers and a pair of high order mode (HOM) couplers to absorb the energy associated to HOM field excited as the bunch passes through the cavity. In the present paper we investigate, using numerical simulations, the impact of the input and HOM couplers on the beam dynamics to zeroth and first order in initial position, and present parametric studies of the strength of these effects for various incoming beam energies. We finally study the impact of this asymmetric field on the beam dynamics, taking as an example the low energy section of the X-ray FEL injector.
\end{abstract}

\section{INTRODUCTION}

In order to study the disturbances associated to the electromagnetic field perturbations induced by the couplers, we used the eigensolver MAFIA. To avoid extensive computer calculation, only the regions of the coupler (including the first of last cell of the cavity) were simulated using a three-dimensional mesh [2]. These 3D simulations produced the electromagnetic field maps $\left\{\mathbf{E}_{u, c}, \mathbf{B}_{u, c}\right\}$ and $\left\{\mathbf{E}_{d, c}, \mathbf{B}_{d, c}\right\}$ respectively for the upstream and downstream coupler areas. The same areas were also simulated without including the couplers and the undisturbed electromagnetic field maps $\left\{\mathbf{E}_{u, 0}, \mathbf{B}_{u, 0}\right\}$ and $\left\{\mathbf{E}_{d, 0}, \mathbf{B}_{d, 0}\right\}$ were generated. From these two sets of simulations the difference fields, i.e. the perturbation of the nominal field due to the effect of the couplers were obtained for the upstream and downstream regions:

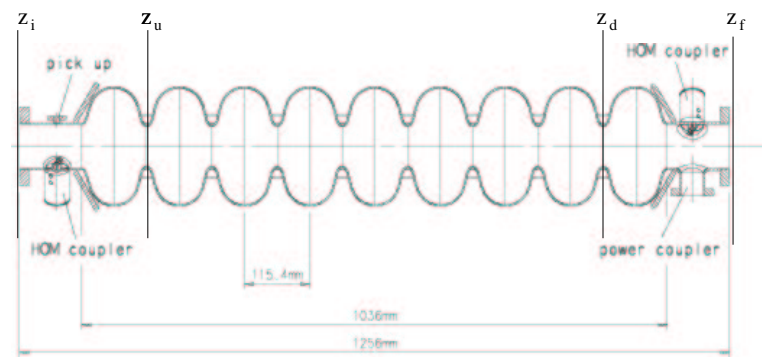

Figure 1: Overview of the TESLA cavity. The area $z_{i}<$ $z<z_{u}$ and $z_{d}<z<z_{f}$ are respectively referred to as upstream and downstream coupler regions.

$$
\begin{array}{r}
\left\{\delta \mathbf{E}_{u}, \delta \mathbf{B}_{u}\right\}=\left\{\mathbf{E}_{u, c}-\mathbf{E}_{u, 0}, \mathbf{B}_{u, c}-\mathbf{B}_{u, 0}\right\}, \text { and } \\
\left\{\delta \mathbf{E}_{d}, \delta \mathbf{B}_{d}\right\}=\left\{\mathbf{E}_{d, c}-\mathbf{E}_{d, 0}, \mathbf{B}_{d, c}-\mathbf{B}_{d, 0}\right\} .
\end{array}
$$

For the upstream region (only one HOM coupler) we have $\mathbf{E}_{u} \in \mathbb{R}$ and $\mathbf{B}_{u} \in \mathbb{I}$ while for the downstream area (HOM and input couplers) $\left\{\mathbf{E}_{d}, \mathbf{B}_{d}\right\} \in \mathbb{C}$. In Figures 2 and 3 we present the electromagnetic difference field map computed for the upstream and downtream regions. Figure 4 is an arrow plot showing the directions of the induced deflection voltage $\left(\mathbf{V}_{\perp} \propto \int_{-\infty}^{+\infty}\left[\mathbf{E}+c \hat{\mathbf{e}}_{z} \times \mathbf{B}\right] d z\right.$.

\section{IMPACT OF COUPLER FIELD ON THE BEAM DYNAMICS}

For the numerical tracking performed with ASTRA, the cavity $\mathrm{TM}_{010-\pi}$ mode is nominally described by its axial E-field, $E_{z}(z)=E_{z}(r=0, z)$, obtained from 2-D MAFIA $(r, z)$ simulations. To study the impact of the coupler on the beam dynamics the difference fields given by Eq. 1 are superimposed to the axial field. The electromagnetic field within the cavity region $\left(z \in\left[z_{i}, z_{f}\right]\right.$, see Fig. 1 for notations) is then described as follows:

$$
\begin{aligned}
\text { if } z_{u}<z<z_{d}, & \\
\mathbf{E}(r, z ; t)= & \widetilde{\mathbf{E}}(r, z) \sin (\omega t+\varphi), \\
\mathbf{B}(r, z ; t)= & \widetilde{\mathbf{B}}(r, z) \cos (\omega t+\varphi), \\
\text { if } z_{i}<z<z_{u}, & \\
\mathbf{E}(x, y, z ; t)= & \left(\widetilde{\mathbf{E}}+\mathcal{R} e\left\{\delta \mathbf{E}_{u}\right\}\right) \sin (\omega t+\varphi), \\
\mathbf{B}(x, y, z ; t)= & \left(\widetilde{\mathbf{B}}+\mathcal{I} m\left\{\delta \mathbf{B}_{u}\right\}\right) \cos (\omega t+\varphi), \\
\text { if } z_{d}<z<z_{f}, & \\
\mathbf{E}(x, y, z ; t)= & \left(\widetilde{\mathbf{E}}+\mathcal{R} e\left\{\delta \mathbf{E}_{d}\right\}\right) \sin (\omega t+\varphi)+ \\
& \mathcal{I} m\left\{\delta \mathbf{E}_{d}\right\} \cos (\omega t+\varphi), \\
\mathbf{B}(x, y, z ; t)= & \left(\widetilde{\mathbf{B}}+\mathcal{I} m\left\{\delta \mathbf{B}_{d}\right\}\right) \cos (\omega t+\varphi)+ \\
& \mathcal{R} e\left\{\delta \mathbf{B}_{d}\right\} \sin (\omega t+\varphi)
\end{aligned}
$$

$\mathcal{I} m$ and $\mathcal{R}$ e respectively refer to the imaginary and real part, $\omega \doteq 2 \pi f$ (where $f$ is the $\mathrm{rf}$ frequency) and $\varphi$ the operating phase. To first order in $r$ we have in cylindrical coordinate $(r, \theta, z) \widetilde{\mathbf{E}}(r, z) \doteq-\frac{r}{2} \frac{d E_{z}}{d z}(z) \hat{\mathbf{e}}_{r}+E_{z}(z) \hat{\mathbf{e}}_{z}$ and $\widetilde{\mathbf{B}}(r, z) \doteq \frac{\omega r}{2 c^{2}} E_{z}(z) \hat{\mathbf{e}}_{\theta}$.

To numerically investigate the focusing property of the cavity, we follow previous work done for the CEBAF-type 

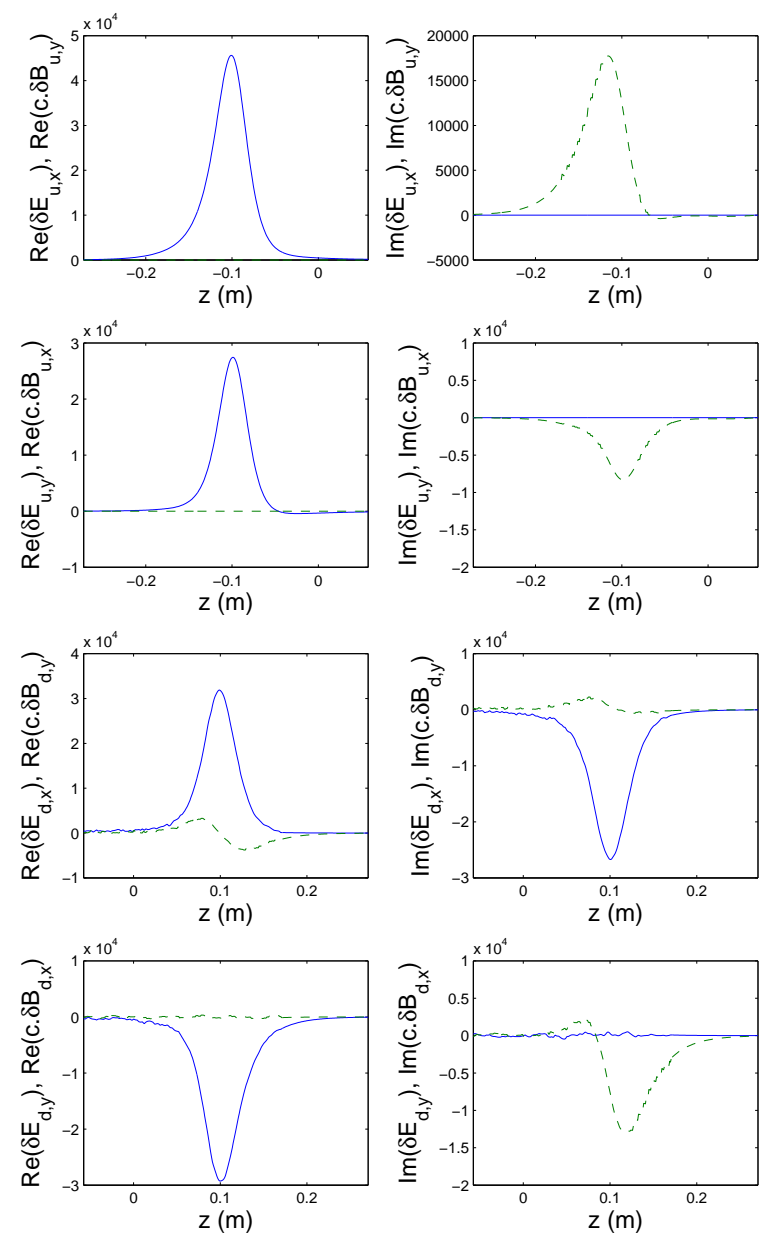

Figure 2: On-axis $(x=y=0)$ transverse fields (in V. $\mathrm{m}^{-1}$ ) versus longitudinal position for the upstream (top four plots) and downstream (bottom four plots) coupler regions. $z=0$ correspond either to the center of the first cell (four top plots) or the center of ninth cell (four bottom plots). The cavity axial field amplitude is $\hat{E}_{z}=50 \mathrm{MV} / \mathrm{m}$.

cavity [3]. Let's consider the changes in transverse momenta of a particle entering the cavity with transverse coordinates $(x=\rho \cos \theta, y=\rho \sin \theta)$ to be of the form

$$
\left[\begin{array}{l}
\Delta P_{x} \\
\Delta P_{x}
\end{array}\right]=\left[\begin{array}{l}
a_{x} \\
a_{y}
\end{array}\right]+\left[\begin{array}{l}
b_{x} x \\
b_{y} y
\end{array}\right]+\left[\begin{array}{l}
c_{x} y \\
c_{y} x
\end{array}\right]
$$

The parameters $a_{x, y}$ characterize the steering effects, $b_{x, y}$ the focusing and $c_{x, y}$ the $x-y$ coupling effects. We consider an initial set of particles arranged on a circle with radius $\rho$ with no longitudinal dimension. Once the distribution has been tracked through the cavity, the coefficients of Eq. 3 can be calculated as

$$
\begin{aligned}
a_{x, y} & =\frac{1}{2 \pi} \int_{0}^{2 \pi} d \theta \Delta P_{x, y} \\
b_{x, y} & =\frac{1}{\rho \pi} \int_{0}^{2 \pi} d \theta\left(\cos \theta \Delta P_{x}, \sin \theta \Delta P_{y}\right)
\end{aligned}
$$
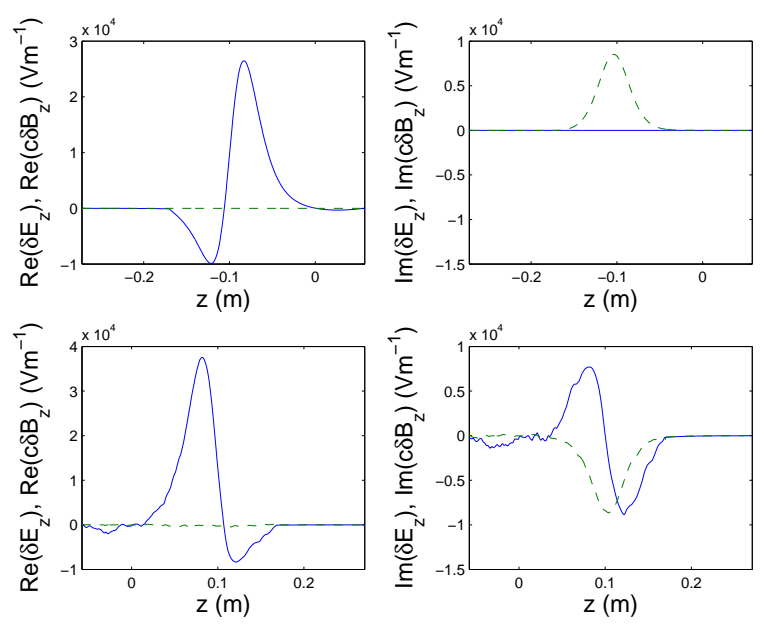

Figure 3: On-axis ( $x=y=0$ ) longitudinal fields (in V.m ${ }^{-1}$ ) versus longitudinal position for the upstream (top two plots) and downstream (bottom two plots) coupler regions. $z=0$ correspond either to the center of the first cell (four two plots) or the center of ninth cell (bottom two plots). The cavity axial field amplitude is $\hat{E}_{z}=50 \mathrm{MV} / \mathrm{m}$.
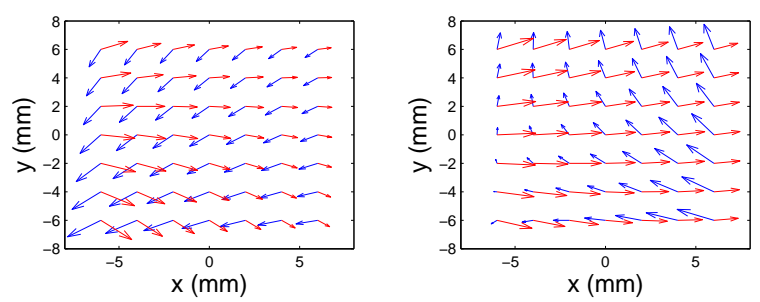

Figure 4: Induced voltage for the upstream (left) and downstream (right) coupler (new geometry $6 \mathrm{~mm}$ ) regions (black: real part; red: imaginary part).

$$
c_{x, y}=\frac{1}{\rho \pi} \int_{0}^{2 \pi} d \theta\left(\sin \theta \Delta P_{x}, \cos \theta \Delta P_{y}\right) .
$$

The dependences of steering and focusing properties on the operating phase of the cavity were investigated. The calculated coefficients of Eq. 3 are presented in Fig. 5 for the case of a TESLA cavity with $\hat{E}_{z} \doteq$ $\max \left[E_{z}(z)\right]=50 \mathrm{MV} / \mathrm{m}$, and incoming beam with energy $\mathrm{E}=1 \mathrm{GeV}$. Note that Eq. 3 can be rewritten

$$
\begin{aligned}
{\left[\begin{array}{l}
\Delta P_{x} \\
\Delta P_{x}
\end{array}\right]=} & {\left[\begin{array}{l}
a_{x} \\
a_{y}
\end{array}\right]+F\left[\begin{array}{l}
x \\
y
\end{array}\right]+} \\
& Q\left[\begin{array}{c}
x \\
-y
\end{array}\right]+S\left[\begin{array}{l}
y \\
x
\end{array}\right]+\kappa\left[\begin{array}{c}
y \\
-x
\end{array}\right],
\end{aligned}
$$

where $\{F, Q\} \doteq \frac{b_{x} \pm b_{y}}{2}$ represent the cylindrical and quadrupole-type focusing, $\{S, \kappa\} \doteq \frac{c_{x} \pm c_{y}}{2}$ account for the skew and solenoidal-type focusing; $S$ and $\kappa$ introduce $x$ $y$ coupling. In our numerical calculations $\kappa$ appears to be the main contributor to $x$-y coupling effects while for the CEBAF-type cavity $S \gg \kappa$ [3]. In Figure 6 we present the values of each of the coefficients of Eq. 5 for different 
cases of incoming beam energies and considering a TESLA cavity with $\hat{E}_{z}=50 \mathrm{MV} / \mathrm{m}$.

\section{IMPACT ON THE XFEL PHOTOINJECTOR}

We present a practical example of the coupler-effects on the beam dynamics by considering the x-ray FEL photoinjector [5]. In the injector, the electron beam is photoemitted in an L-band $(f=1.3 \mathrm{GHz})$ rf-gun and directly injected into a standard TESLA-type accelerating module composed of eight cavities and accelerated to $\sim 190 \mathrm{MeV}$. The first two cavities of the accelerating module are operated with $\hat{E}_{z}=23 \mathrm{MV} / \mathrm{m}$ and the remaining six cavities with $\hat{E}_{z}=50 \mathrm{MV} / \mathrm{m}$. Figure 7 compares the beam parameter evolutions along the injector beamline from the photocathode surface $(z=0)$ up to the end of the first accelerating module. The field disturbances caused by the coupler result in a $16 \%$ and $1 \%$ relative emittance growth respectively in the horizontal and vertical plane. The induced steering results in beam displacements at the accelerating module exit of $|\delta x|=680 \mu \mathrm{m}$ and $|\delta y|=170 \mu \mathrm{m}$, and no significant $x-y$ coupling is observed.

\section{SUMMARY}

We have presented a technique to include the effects of input and HOM couplers in the beam dynamics calculations. The impact of the field disturbances was characterized in term of dipole and focusing strengths. A simulation of the X-ray FEL injector indicates the impact of the couplers on the beam parameters is small. We are currently exploring the impacts of couplers on the beam dynamics in the ILC main linac.
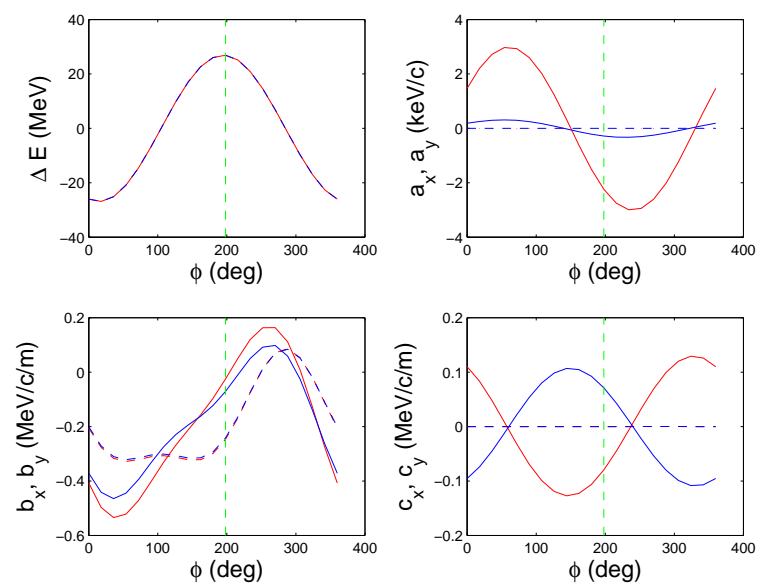

Figure 5: Calculated dipole, focusing strength (as defined in Eq. 3) with (solid) and without (dashed) including the coupler field disturbance. blue: $x$-axis; red: $y$-axis. The vertical dashed green line represents the phase corresponding to maximum energy gain.
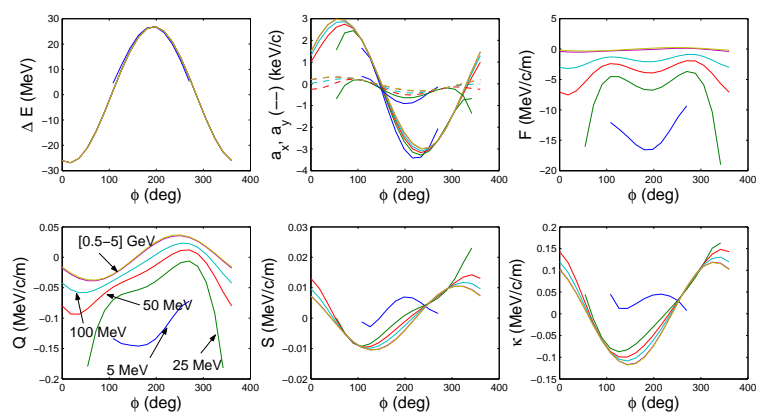

Figure 6: Calculated energy gain $(\Delta E)$, steering $\left(a_{x}, a_{y}\right)$, focusing $(F, Q)$ and coupling $(S, \kappa)$ coefficients of Eq. 5. The calculations are performed for various incoming beam energies $\mathcal{E}=[0.005,0.025,0.05,0.1,1,5] \mathrm{GeV}$
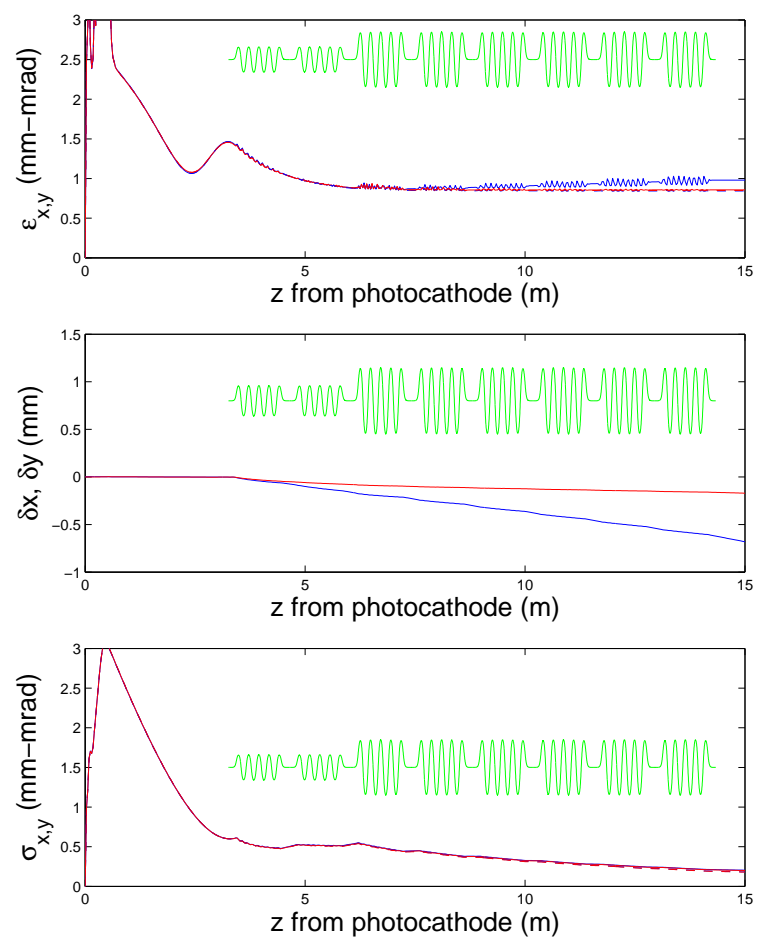

Figure 7: Simulated normalized transverse emittance(top plot), beam position (middle plot), and beam rms sizes (bottom plot) evolution along the x-ray FEL photoinjector with (solid line) and without (dashed line) including the coupler effects. red: vertical plane; blue: horizontal plane. The linac phase is chosen for maximum energy gain. The green curves represent the axial electric field in the cavity in arbitrary units.

\section{REFERENCES}

[1] B. Aune et al., Phys. Rev. ST Accel. Beams 3,092001 (2000)

[2] M. Dohlus et al., proceedings of EPAC 2000, p. 2096 (2000)

[3] Z. Li, et al., proceedings of PAC'93, p. 179 (1993)

[4] K. Flöttmann, Astra user manual (2001); available at http://www.desy.de/rmpyflo/Astra_Dokumentation

[5] K. Flöttmann, et al., proceedings of PAC 2001, p. 2236 (2001) 